\title{
Classifying rational densities using two one-dimensional cellular automata
}

\author{
H. F. Chau, * K. K. Yan, K. Y. Wan, and L. W. Siu \\ Department of Physics, University of Hong Kong, Pokfulam Road, Hong Kong
}

(Received 9 September 1997)

\begin{abstract}
Given a (finite but arbitrarily long) string of zeros and ones, we report a way to determine if the number of ones is less than, greater than, or equal to a prescribed number by applying two sets of cellular automaton rules in succession. Thus, we solve the general one-dimensional density classification problem using two cellular automata. [S1063-651X(98)07402-9]
\end{abstract}

PACS number(s): 05.50.+q, 05.70.Fh, 89.80.+h

\section{INTRODUCTION}

Cellular automaton (CA) is an extremely simple local interaction model of natural systems $[1,2]$. In this paper, we restrict ourselves to considering a conventional definition of $\mathrm{CA}$, namely, that both the number of possible states at each site and the number of CA rules in the rule table are finite. That is to say, the state of each site in the next time step depends only on a finite number of neighboring sites in the system. Although the rules of CA are simple and local, complicated spatial patterns can be formed. Moreover, from the computer science point of view, CA can be regarded as a very special kind of Turing machine with no internal memory. In fact, tailor-made CA can be used to simulate certain logical operations [3]. Therefore, it is instructive to know the power and limitations of $\mathrm{CA}$ in computation.

Since CA is essentially a special Turing machine with no internal memory, it is natural to ask if CA can be used to perform certain tasks that require global counters. An example is the so-called density classification problem. Consider a one-dimensional (finite but arbitrarily long) chain of sites with periodic boundary condition. Each site is either in state zero or state one. The problem is to change the state of every site to zero if the number of zeros is more than the number of ones in the chain. Otherwise, every site is set to state one. Clearly, the density classification problem is trivial if one can invoke an internal memory to count the number of zeros in the chain. Alternatively, one can solve this problem if the rule table scales with the system size $N$ so that the CA model becomes nonlocal. However, Land and Belew showed that density classification cannot be done perfectly using a single one-dimensional CA [4].

Later on, Fukś pointed out that the density classification problem in fact can be solved if we apply two CA rules in succession [5]. For a one-dimensional lattice of $N$ sites, he first applies the Wolfram elementary CA rule 184 (the socalled traffic rule [6]) $\lfloor(N-2) / 2\rfloor$ times. Then, he applies the Wolfram elementary CA rule 232 (the so-called majority rule) $\lfloor(N-1) / 2\rfloor$ times. The combined automaton solves the density classification problem succinctly [5].

With slight modifications, Fukś is able to classify density in the form $1 / n$ for some integer $n \geqslant 2$ [5]. Then, Fukś went on to ask if it is possible to use combined CA to classify an

\footnotetext{
*Electronic address: hfchau@hkusua.hku.hk
}

arbitrary density $\rho_{c}$. Here, we report a simple solution to his problem using a combination of two CAs provided that $\rho_{c}$ is a rational number. But before stating our CA rules, let us restate the density classification problem in the most general context.

First, let us define the problem in a more precise way. We are given a critical density $\rho_{c}$ between zero and one. Then we consider a one-dimensional lattice made up of $N$ sites with periodic boundary condition. (We can always assume that $N$ is sufficiently large to avoid trivial cases.) The state of each site can be either zero or one. And the initial density of ones in the system is denoted by $\rho$. (That is, there are $N \rho$ sites in the system with states equal to one.) Our goal is to find a combination of two CA rules (with finite rule tables) such that after going through the $\mathrm{CA}$, the final states of the sites on the lattice all become zeros (and all ones) if $\rho<\rho_{c}$ (and $\rho>\rho_{c}$, respectively). And in the case of $\rho=\rho_{c}$, the final state consists of exactly $N \rho_{c}$ sites with states equal to one. (From the nonlinear-dynamics point of view, all the zeros and all the ones are the two stable fixed points of the CA dynamics, whereas the $a$ density equals to $\rho_{c}$ is an unstable fix point of the CA dynamics.) In addition, the two CA rules are independent of the lattice size $N$ for sufficiently large $N$. (Thus, our two CA rules are truly local.)

Since the number of sites $N$ is finite, it suffices for us to consider the case where $\rho_{c}$ is a rational number written in the form $p / q$ for some relatively prime integers $p$ and $q$. Furthermore, we can also assume that $p \neq 0$ and $p \neq q$, since classifying $\rho_{c}=0$ and 1 are trivial using CA. Now we report the two CA rules we use to solve the density classification problem for rational critical density.

\section{MODIFIED TRAFFIC RULE}

Our generalized traffic rule goes as follows. First, we regard a site with state one as being occupied by a car. Otherwise, that site is empty. Then a car can move in the next time step to the right by one site if and only if (a) its immediate right-neighboring site is unoccupied and (b) the $q-1$ consecutive right-neighboring sites are occupied by at most $p$ -1 cars. Otherwise, the car stays in its original position. For example, if $\rho_{c}=2 / 5$, then the first cars in the sequences 10000 and 10100 can move one step forward in the next time step, while the first cars in the sequences 11000 and 10011 cannot. Readers can readily verify that the total number of cars in the system is conserved under the above set of rules. 
Moreover, one can easily convert the above set of traffic rules into a finite CA rule table consisting of $2^{q+1}$ rules. In addition, when $\rho_{c}=1 / 2$, the modified traffic rule is reduced to Wolfram's elementary CA rule 184 .

We define the local car density at each site to be the total number of cars contained in that site and in its $q-1$ immediate right-neighboring sites divided by $q$. Thus, the local car density of a site changes when and only when a car enters that site or a car leaves the $(q-1)$ th site to its right. For simplicity, a collection of sites is said to be in a low-density region if the local car density of each site in the collection is less than or equal to $\rho_{c}$. And a high-density region is defined as a collection of sites with local car densities greater than $\rho_{c}$. Now, we prove two theorems concerning the distribution of high- and low-density regions under the repeated actions of the modified traffic rules.

Lemma 1. If the initial density $\rho$ of the system is less than or equal to $\rho_{c}$, then after at most $\lceil N(\max (q, 2 p)-1) / q\rceil+q$ -2 time steps, the local car density for every site is less than or equal to $\rho_{c}$.

Proof. Consider a collection of sites with local car density greater than $\rho_{c}$ in the initial system configuration. Using the modified traffic rule, in the co-moving frame of a car, no site will have a car density exceeding $(p+1) / q$ if that car is originally located at a site with a local car density less than or equal to $\rho_{c}$. In addition, such an aggregation of cars above the density threshold $\rho_{c}$ will be achieved within the first $q-1$ time steps. Since the overall car density of the system is less than or equal to $\rho_{c}$, upon repeated applications of the modified traffic rules, cars will gradually move out of local regions with a car density exceeding $\rho_{c}$ (if any). Note that the local car densities of those cars moving out of these "high-density regions" are less than or equal to $\rho_{c}$. Moreover, once these cars are "dissolved" from a local highdensity region, the local car density of the sites containing these cars will never exceed $\rho_{c}$ unless these cars merge into a high-car-density region in front.

Suppose none of the cars moving out of a high-cardensity region will be stopped by another high-car-density region in front. Then, once all the high-car-density regions dissolve completely, no further high-density region will be formed thereafter. Thus, our assertion that all sites will have a local car density not exceeding $\rho_{c}$ asymptotically is true in this case. So, it remains to consider the case in which some of the cars moving out of a high-car-density region move into (and hence are temporarily stopped by) a high-cardensity region in front. Since the overall car density of the entire system does not exceed $\rho_{c}$, at least one of the highcar-density regions will start to dissolve. Note that $N$ is finite, and those cars merging into a high-density region will readily redissolve once they are allowed to move. After a finite number of time steps, all the high-density regions will disappear. After that, no more high-density regions can form. Thus, our assertion that all sites will have local car density not exceeding $\rho_{c}$ is also true is this case.

Finally, we estimate the number of time steps required to reach this state. Since the updating is taken in parallel, the worst case occurs when some of the local high-density regions are formed during the first $q-2$ time steps. Then all the cars merge into a single high-car-density region before they finally dissolve. According to the modified traffic rules, if $p \leqslant q / 2$, then all cars dissolved from a high-density region will not be blocked. In this case, it takes at most $q-1$ time steps to dissolve $p$ cars from a high-density region. On the other hand, if $p>q / 2$, then some of the dissolved cars will still be blocked occasionally. And it takes $q-1+(2 p-q)$ $=2 p-1$ time steps to dissolve $p$ cars from a high-density region. Therefore, in any case, it requires at most $\lceil N \rho(\max (q, 2 p)-1) / p\rceil \leqslant\lceil N(\max (q, 2 p)-1) / q\rceil$ time steps to completely dissolve a high-density region made up of $N \rho$ cars. Hence, our assertion is proved.

Lemma 2. If the initial density $\rho$ of the system is greater than $\rho_{c}$, then after at most $\lceil N(q-p)(\max (q, 2 p)-1) / p q\rceil$ time steps, the local car density for every site is greater than or equal to $\rho_{c}$.

Proof. We may assume that the local car density at each site initially is less than or equal to $\rho_{c}$. Otherwise, no car can move to the right at the beginning and our assertion is trivially true. Since the overall car density $\rho$ is greater than $\rho_{c}$, all the low-car-density regions (sites with local car density less than $\rho_{c}$ ) are surrounded by high-car-density ones (sites with car density greater than or equal to $\rho_{c}$ ). Thus, cars can gradually move from the high-density to the low-density regions. And using similar arguments as in Lemma 1, after a finite number of time steps, the entire system is contained in a single high-density region. And from that time on, no car in the system can move.

We move on to estimate the number of time steps required to reach this "frozen" state. Similar to the argument in Lemma 1, the worst case occurs when there is only one low-density region initially. And it is easy to verify that the number of time steps required to reach a frozen state for such an initial system configuration equals $\lceil N(1-\rho)(\max (q, 2 p)$ $-1) / p\rceil \leqslant\lceil N(q-p)(\max (q, 2 p)-1) / p q\rceil$.

Combining Lemmas 1 and 2, we conclude the following.

Theorem 1. By applying the modified traffic rules for $\lceil N(\max (q, 2 p)-1) \max (q-p, p) / p q\rceil+q-2$ times, an initial system configuration will be segregated into one of the following three cases.

(a) If $\rho<\rho_{c}$, then the local car density at every site is less than or equal to $\rho_{c}$. In addition, at least one of the sites will have local car density strictly less than $\rho_{c}$.

(b) If $\rho=\rho_{c}$, then the local car density at every site equals $\rho_{c}$.

(c) If $\rho>\rho_{c}$, then the local car density at every site is greater than or equal to $\rho_{c}$. In addition, at least one of the sites will have a local car density strictly greater than $\rho_{c}$.

\section{MODIFIED MAJORITY RULE}

After segregating the system configuration according to its initial density $\rho$, the density classification problem becomes straightforward. We consider the following modified majority rule for a given critical density $\rho_{c} \equiv p / q$. The state of a site in the next time step is one if there are at least $2 p$ +1 ones in the $2 q+1$ sites consisting of itself and the $q$ consecutive left- and right-neighboring sites. Otherwise, the state of this site in the next time step is zero. For example, if $\rho_{c}=1 / 2$, then the states of the middle sites in the next time step for 10101 and 01010 are one and zero, respectively. Clearly, the modified majority rule is local.

Now, we present the results of applying the modified ma- 
jority rule to certain system configurations which are of interest.

Lemma 3. Any system configuration where the local car density at every site equals $\rho_{c}$ is a fixed point of the modified majority rule dynamics.

Proof. Consider an arbitrary site $\alpha$ in the system. Since the local car density at every site equals $\rho_{c}$, there are precisely $2 p$ sites in state one among the $2 q$ neighboring sites of $\alpha$. Thus, the majority rule implies that the state of site $\alpha$ in the next time step is equal to its present state. Consequently, this system configuration is a fixed point of the modified majority rule.

Lemma 4. Suppose the local car density at every site is greater than or equal to $\rho_{c}$ and also that the total density of the system is strictly greater than $\rho_{c}$. After applying the modified traffic rule $[N / 2(q-1)]$ times, the state of every site in the system will become one. Similarly, if the local car density at every site is less than or equal to $\rho_{c}$ and also if the total density of the system is strictly less than $\rho_{c}$, then after applying the modified traffic rule $[N / 2(q-1)]$ times, the state of every site in the system will become zero.

Proof. For simplicity, we only consider the case in which $\rho>\rho_{c}$. The proof for the case in which $\rho<\rho_{c}$ is similar. From Lemma 3 , we know that the local car density at every site must be greater than or equal to $\rho_{c}$ after repeated applications of the modified majority rule. Moreover, if the local car density of a site $\alpha$ exceeds $\rho_{c}$, then the states of $\alpha$ and its $q-1$ left- and right-neighboring sites must be one in the next (and hence all subsequent) time step. In other words, the propagation speed for state one is $q-1$ sites per time step both leftward and rightward. Therefore, after $\lceil N / 2(q-1)\rceil$ time steps, all sites in the system will be in state one. Hence, our assertion is proved.

Combining Theorem 1, Lemma 3, and Lemma 4 with the fact that the size of the rule tables for both the modified traffic and the modified majority rules are independent of $N$, we obtain the following CA density classification theorem:

Theorem 2 (Density Classification By CA). Let $\rho_{c}=p / q$ be a rational number between zero and one, with $p$ and $q$ being relatively prime positive integers. Then the density classification problem can be solved using the following two CA rules: apply the modified traffic rules $\lceil N(\max (q, 2 p)$ -1) $\max (q-p, p) / p q\rceil+q-2$ times and then the modified majority rule $\lceil N / 2(q-1)\rceil$ times.

\section{DISCUSSION}

In summary, we report a way to classify the density of ones using two one-dimensional binary CAs provided that the density threshold $\rho_{c}$ is a rational number. Our result, therefore, generalizes that of Fukś [5]. Besides, our construction also takes into account the case where the density $\rho$ is equal to its critical value $\rho_{c}$-something Fukś does not consider seriously. Because of the local nature of CA rules, we believe that the time complexity of a general density classification problem using any combination of CA rules is at least $\mathrm{O}(N)$. Thus, apart from a constant speedup, our set of density classification CA rules is probably the least timeconsuming.

As we have discussed in Sec. I, if the number of sites in the system $N$ is finite, it suffices to restrict ourselves to considering rational density classification. Any irrational density can be well approximated by a corresponding rational density. Actually, by approximating an irrational density by better and better rational numbers, the size of the $\mathrm{CA}$ rule table grows. Thus, we believe that combinations of $\mathrm{CA}$ rules are unlikely to be powerful enough to classify irrational density in an infinite one-dimensional system.

\section{ACKNOWLEDGMENT}

This work was supported by the University of Hong Kong CRCG grant under Contract No. 335/025/0040.
[1] See, for example, S. Wolfram, Cellular Automata and Complexity (Addison-Wesley, Reading, MA, 1994).

[2] See, for example, T. Toffoli, in Encyclopedia of Physics, edited by R. G. Lerner and G. L. Trigg, 2nd ed. (VCH Publishers, New York, 1990), p. 126 for a definition of cellular au- tomaton.

[3] A. N. Korotkov, Appl. Phys. Lett. 67, 2412 (1995).

[4] M. Land and R. K. Belew, Phys. Rev. Lett. 74, 5148 (1995).

[5] H. Fukś, Phys. Rev. E 55, 2081 (1997).

[6] M. Fukui and Y. Ishibashi, J. Phys. Soc. Jpn. 65, 1868 (1996). 